\section{POS0031 THE PREVALENCE OF INFLAMMATORY BACK PAIN AND HLA-B27 IN A LARGE POPULATION-BASED COHORT IN THE NETHERLANDS}

S. Kieskamp ${ }^{1}$, S. Arends ${ }^{1}$, E. Brouwer ${ }^{1}$, H. Bootsma ${ }^{1}$, I. M. Nolte ${ }^{2}$, A. Spoorenberg ${ }^{1}$. 'University Medical Center Groningen, Rheumatology and Clinical Immunology, Groningen, Netherlands; ${ }^{2}$ University Medical Center Groningen, Department of Epidemiology, Groningen, Netherlands

Background: Although chronic low back pain ( $\geq 3$ months) before the age of 45 and inflammatory back pain (IBP) are regarded as early presenting and key features of axial spondyloarthritis (axSpA), and Magnetic Resonance Imaging (MRI) can be used to demonstrate sacroiliitis, the substantial delay in the diagnosis of axSpA has not improved.(1) Additionally, knowledge on the prevalence of chronic low back pain before the age of 45 and IBP in combination with the axSpA-related genetic risk factor Human Leukocyte Antigen-B27 (HLA-B27) in the general population is scarce.

Objectives: To estimate the prevalence of chronic low back pain before the age of 45 and IBP in combination with the presence of HLA-B27 in a large Dutch population based cohort.

Methods: Participants of the Lifelines cohort, a large population-based cohort of the northern region of the Netherlands, filled out a questionnaire on chronic low back pain and IBP. Chronic low back pain was defined as an affirmative answer to the question 'Did you suffer from low back pain for $\geq 3$ months?'. IBP was questioned based on the validated European Spondyloarthropathy Study Group (ESSG) IBP criteria and was confirmed if at least 4 out of the following 5 criteria were present: (a) onset before age 45, (b) insidious onset, (c) improvement with exercise, (d) associated with morning stiffness, (e) at least 3 months duration. Participants reporting to have been diagnosed with axSpA were identified using variations of the search terms "Bechterew", "spondyloarthritis" and "ankylosing spondylitis". The Illumina global screening array (GSA) beadchip-24 v1.0 was used to genotype genome-wide SNPs in a subset of Lifelines participants. HLA-B haplotypes were imputed using neighboring SNPs with HIBAG, which is an R-package, using published parameter estimates.(2) The predicted HLA-B haplotype was considered valid if the posterior probability was $>80 \%$.

Results: In total 94,277 Lifelines participants answered the chronic low back pain question, of which $93,665(99.4 \%)$ completed the ESSG IBP questions. Of these participants, $56,008(59.8 \%)$ were female, mean age was $45.6 \pm 12.8$ years and 22,192 (23.7\%) reported to have been suffering from chronic low back pain. In this chronic low back pain group, the pain began before the age of 45 in $17,122(77.2 \% ; 18,3 \%$ of entire Lifelines population) participants, and 13,514 (60.9\%; $14.4 \%$ of entire Lifelines population) participants reported to have IBP according to the ESSG criteria.

Of 32,785 participants genetic data were available and in $29,399(89.7 \%)$ the HLA-B haplotype could be determined with high prediction accuracy, of which 2,279 $(7.8 \%)$ participants were HLA-B27 positive. Of these HLAB27 positive participants, 1,610 (70.6\%) also had available chronic low back pain data, of which $373(23.2 \%)$ reported chronic low back pain. Of these 373 patients with chronic back pain and HLA-B27 positivity, the pain began before the age of 45 in $296(79.4 \%)$, and 237 (64.2\%) fulfilled the ESSG IBP criteria of which only $11(4.6 \%)$ participants reported to be diagnosed with axSpA.

Conclusion: In this large population-based cohort, $18.3 \%$ of participants reported chronic low back pain that began before the age of $45.14 .4 \%$ of the participants reported IBP, which is relatively high in comparison to previous studies. HLA-B27 prevalence (7.8\%) was similar to previously published data from the North-Western European population. The vast majority of participants with both IBP and the presence of HLA-B27 did not report an axSpA diagnosis. A next step in the analyses will be to explore associations with other demographic and clinical factors present including additional SpA features.

REFERENCES:

[1] Zhao SS, et al. Rheumatology (Oxford). 2021; keaa807

[2] Internet: https://zhengxwen.github.io/HIBAG/hibag_index.html (Accessed: 25 November 2020)

Disclosure of Interests: Stan Kieskamp: None declared, Suzanne Arends Grant/research support from: Research support from Pfizer, Elisabeth Brouwer Speakers bureau: Roche, Consultant of: Roche, Hendrika Bootsma Grant/ research support from: Roche, Ilja M. Nolte: None declared, Anneke Spoorenberg Consultant of: Abbvie, Pfizer, MSD, UCB, Novartis, Grant/research support from: Abbvie, Pfizer, UCB, Novartis.

DOI: 10.1136/annrheumdis-2021-eular.3988

\section{Imaging in axial spondyloarthritis}

\begin{tabular}{l|l}
\hline POSO032 & SCORING MRI STRUCTURAL LESIONS IN \\
SACROILIAC JOINTS OF PATIENTS WITH AXIAL \\
SPONDYLOARTHRITIS: HOW MANY SLICES ARE \\
OPTIMAL?
\end{tabular}

W. P. Maksymowych ${ }^{1,2}$, U. Weber ${ }^{3}$, X. Baraliakos ${ }^{4}$, P. Machado ${ }^{5}$, S. Juh Pedersen $^{6,7}$, J. Sieper $^{8}$, S. Wichuk ${ }^{1}$, D. Poddubnyy ${ }^{8}$, M. Rudwaleit ${ }^{9}$, D. Van der Heijde ${ }^{10}$, R. B. M. Landewé ${ }^{11}$, J. Paschke ${ }^{2}$, M. Østergaard ${ }^{6,7}$, R. G. Lambert ${ }^{12}$ on behalf of ASAS MRImagine. ${ }^{1}$ University of Alberta, Medicine, Edmonton, Canada; ${ }^{2}$ CARE Arthritis, Rheumatology, Edmonton, Canada; ${ }^{3}$ Practice Buchsbaum, Medicine, Schaffhausen, Switzerland; ${ }^{4}$ Rheumazentrum Ruhrgebiet, Rheumatology, Herne, Germany; ${ }^{5}$ University College London, Rheumatology, London, United Kingdom; ${ }^{6}$ COPECARE, Rheumatology, Copenhagen, Denmark; ${ }^{7}$ Rigshospitalet, Clinical Medicine, Glostrup, Denmark; ${ }^{8}$ Charité Universitätsmedizin, Rheumatology, Berlin, Germany; ${ }^{9}$ Klinikum Bielefeld, Rheumatology, Bielefeld, Germany; ${ }^{10}$ Leiden University Medical Centre, Rheumatology, Leiden, Netherlands; ${ }^{11}$ Academic Medical Center, Rheumatology, Amsterdam, Netherlands; ${ }^{12}$ University of Alberta, Radiology and Diagnostic Imaging, Edmonton, Canada

Background: There is no international consensus on the optimal number of slices for evaluation of MRI structural lesions in the SIJ. An "all slice" method evaluates lesions from the most anterior slice, defined as the first slice with vertical height of $\geq 1 \mathrm{~cm}$ of the SIJ joint cavity, up to the most posterior slice, defined as the most posterior slice where $\geq 1 \mathrm{~cm}$ vertical height of the cartilaginous portion is still visible. The SPARCC method scores the transitional slice between cartilaginous and ligamentous compartments as the first slice and then an additional 4 slices anterior to the transitional slice.

Objectives: We aimed to investigate inter-reader reliability, the extent of detection of lesions, and frequency of cases with a positive MRI for structural lesions when using an "all slice" approach versus the SPARCC scoring of 5 central slices.

Methods: MRI T1W images with DICOM series were available from 148 cases who had MRI performed in the ASAS-Classification Cohort. Seven central readers recorded MRI lesions in an eCRF that recorded global assessments of presence/absence of changes suggestive of axSpA and structural lesions typical of axSpA. Structural lesions per the ASAS definitions were also recorded in consecutive semicoronal slices using the "all slice" approach, but also recording the transitional slice, according to their presence/absence in SIJ quadrants (erosion, fat lesion, sclerosis) or halves (backfill, ankylosis). Structural lesion frequencies were assessed descriptively according to majority agreement $(\geq 4 / 7)$ of central readers and also any 2 central readers. Reliability for detection of MRI lesions was compared between central and local readers using the ICC.

Results: The mean (SD) (range) number of anterior and posterior slices peripheral to the 5 central slices was $1.0(1.0)(0-4)$ and $2.2(1.8)(0-6)$ per case, respectively. There were 2 cases $(1.4 \%)$ where $\geq 2$ readers scored structural lesions in peripheral slices but not in the 5 central slices. The mean percentage of the total structural lesion score that was captured by the 5 central slices was $>75 \%$ for all types of lesions except ankylosis $(59 \%)$ (Table 1). Inter-reader reliability was greater for all lesions when assessing the 5 central slices and especially for erosion and backfil (Table 1).

Conclusion: The major component of structural lesion data is captured by assessment of 5 slices, which includes the transitional slice and the subsequent 4 anterior slices. Moreover, reliability for detection of structural lesions is substantially worse in peripheral slices.

Disclosure of Interests: None declared.

DOI: 10.1136/annrheumdis-2021-eular.3427 